\title{
Collisionless reversed magnetic shear trapped electron instability and contribution of sidebands to anomalous transport
}

\author{
André L. Rogister ${ }^{\mathrm{a})}$ \\ Institut für Plasmaphysik, Forschungszentrum Jülich Gesellschaft mit beschränkter Haftung (GmbH), \\ Trilateral Euregio Cluster, European Atomic Energy Community (EURATOM) Association, \\ D-52425 Jülich, Germany \\ Raghvendra Singh \\ Institute for Plasma Research, Bhat, Gandhinagar 382 428, India
}

(Received 4 August 2005; accepted 13 October 2005; published online 22 November 2005)

By keeping account of the trapped electron $\nabla B$ and curvature drifts, it is found that the spatial decay of the collisionless electron drift wave is governed either by the trapped electron response or by the resonant interaction of ions with the sidebands of the primary oscillation. In the former case, pairs of spatially bounded unstable and damped solutions are obtained for negative magnetic shear $(\hat{s}$ $<0)$ if, as usual, $L_{T_{e}}=1 / \partial_{r} \ln T_{e}<0$; there are no bounded solutions if $\hat{s} L_{T_{e}}<0$. In the latter case, there is either a set of bounded damped solutions if $\eta_{i}>0$ or a set of bounded unstable solutions if $\eta_{i}<0$. The unstable modes have a radiating character and the growth rates are $\gamma \sim(2 n$ $+1) \sqrt{1+2 q^{2}}|\hat{s}|\left|L_{N} \omega_{e}^{*} / q R\right|$ ( $n$ is the Hermite polynomial solution index, $q$ the safety factor, $\hat{s}$ the magnetic shear parameter, $R$ the major radius, $\omega_{e}^{*}$ the electron diamagnetic frequency, $L_{N}$ $=1 / \partial_{r} \ln N_{e}$, and $\left.\eta_{i}=L_{N} / L_{T_{i}}\right)$. The sidebands are responsible for unusually large ratios $Q_{e} / T_{e} \Gamma_{e}$, where $Q_{e}$ and $\Gamma_{e}$ are the anomalous electron energy flux and the particle flux. These results may explain the box-type $T_{e}$ profile observed in lower hybrid current drive reversed magnetic shear plasmas on the Japan Atomic Energy Research Institute Tokamak 60 Upgrade (JT-60U) [H. Ninomiya and the JT-60U Team, Phys. Fluids B 4, 2070 (1992)]. It is finally demonstrated that the ballooning hypothesis generally leads to conflicting requirements: it is thus hardly relevant for the electron drift branch! The "radiating" boundary condition that has formerly been imposed on the slab solution is finally discussed. (C) 2005 American Institute of Physics. [DOI: 10.1063/1.2134770]

\section{INTRODUCTION}

In microinstability theory, the nonadiabatic trapped electron response has always been considered as a perturbation and thus estimated in leading order only. Considering the electron drift branch, this is justified if $\sqrt{2 \epsilon} \omega_{e}^{*} / \nu_{e, \text { eff }}<k_{r}^{2} a_{s}^{2}$ $<1$, where the left-hand side is a measure of the nonadiabatic trapped electron response for $\nu_{e, \text { eff }} \gg \omega_{e}^{*}$ and the righthand side characterizes the radial eigenvalue operator $(\epsilon$ $=r / R$ is the inverse aspect ratio, $r$ and $R$ are the minor and major radii, $\omega_{e}^{*}$ is the electron diamagnetic frequency, $\nu_{e, \text { eff }}$ $=\nu_{e} / \epsilon$, where $\nu_{e}$ is the electron collision frequency, $k_{r}$ is the radial wave vector, $a_{s}=c_{s} / \Omega_{i}, c_{s}=\sqrt{T_{e} / m_{i}}$ is the sound velocity, and $\Omega_{i}$ is the ion gyrofrequency). In other cases, the full trapped electron response must be considered, including the effect of the $\nabla B$ and curvature drifts. In this paper, the ratio of the equilibrium densities of trapped and passing electrons $\left(N_{e}\right)_{\text {trap }} /\left(N_{e}\right)_{\text {pass }} \equiv \sqrt{\epsilon(1+\cos \theta)} /[1-\sqrt{\epsilon(1+\cos \theta)}]$ is considered to be of order unity [The magnetic field is $B=B_{0} /(1$ $+\epsilon \cos \theta)$ where $\theta=0$ corresponds to the outboard midplane of the axisymmetric toroidal plasma].

A priori, two limiting cases must be envisaged: the quasislab limit, corresponding to $k_{r} \Delta_{l}>1$, and the ballooning limit, corresponding to $k_{r} \Delta_{l}<1$; here, $\Delta_{l}=-1 / l \partial_{r} q$ is the distance between neighboring rational surfaces, $l(m)$ the toroi-

\footnotetext{
${ }^{a)}$ Electronic mail: a.rogister@fz-juelich.de
}

dal (poloidal) mode number, and $q$ the safety factor. Only the quasislab electron drift branch is considered hereafter as it will be demonstrated on the basis of earlier works that the ballooning hypothesis generally leads to conflicting requirements and is therefore hardly relevant.

Sidebands $(m \pm 1, l)$ of the primary perturbation centered on the rational surface $q(r)=-m / l$ are introduced by the $\epsilon \cos \theta$ modulation of the magnetic field, mainly via the ion $\nabla B$ and curvature drifts. The parallel wave vector of the sidebands, $1 / q R$, is larger than that of the primary perturbation, $1 / k_{r} \Delta_{l} q R$. Resonant interaction of ions with the sidebands thus prevails over resonant interaction with the primary oscillation. Two cases can occur.

(i) Ion resonant interaction with the sidebands dominates over the collisionless trapped electron response: here, the Landau residue leads to either spatially bounded damped solutions if $\eta_{i}>0$ or spatially bounded unstable ones if $\eta_{i}<0 \quad\left(\eta_{i}\right.$ $=\partial_{r} \ln T_{i} / \partial_{r} \ln N_{i}$ and we assume $N_{i}=N_{e}$ ).

(ii) The trapped electron curvature terms are dominant: we find here two spatially bounded solutions, viz., an unstable and a damped one if $\hat{s} L_{T_{e}}>0$, and no bounded solution whatever if $\hat{s} L_{T_{e}}<0 \quad\left(\hat{s}=r \partial_{r} \ln q\right.$ is the magnetic shear parameter); since $L_{T_{e}}$ $=T_{e} / \partial_{r} T_{e}$ is usually negative, instability requires negative magnetic shear. 
In both cases the unstable solutions have an "outgoing" or "radiating" character and their growth rate is proportional to the magnetic shear parameter:

$$
\gamma_{l, n}=\Im m \omega_{l}= \pm(2 n+1) \sqrt{1+2 q^{2}} \frac{\left|L_{N}\right|}{\left|L_{S}\right|} \frac{\alpha_{i} \omega_{e, l}^{*}}{1-\left\langle\left(f_{e}\right)_{\text {trap }}\right\rangle_{\theta}},
$$

where $n$ is the Hermite polynomial solution index, $\left\langle\left(f_{e}\right)_{\text {trap }}\right\rangle_{\theta}$ the normalized flux surface averaged trapped electron density, $L_{N}=1 / \partial_{r} \ln N_{e}, \alpha_{i}=1+\tau_{i}\left(1+\eta_{i}\right)$, and $\tau_{i}=T_{i} / T_{e}$. This result leads us to comment on the actuality of magnetic shear damping in cylindrical plasmas.

The quasislab ion dynamics has been considered in Refs. 1 and 2 ; in the present paper, the corresponding calculation will thus be presented briefly. However, the effect of the sidebands on anomalous transport has not been investigated in the literature. (The ballooning transformation is most often applied $a b$ initio!) It is found that they are responsible for large values of the ratio $Q_{e} / T_{e} \Gamma_{e}$, where $Q_{e}$ and $\Gamma_{e}$ are the anomalous electron energy flux and particle flux, and thus to large diffusive versus convective electron heat transport.

The paper is organized as follows. The linear electron gyrokinetic equation and a suitable ordering of the parameters are introduced in Sec. II. The quasislab electron drift branch solution is derived by expansion in Sec. III, leading to the radial eigenvalue equation. The eigenfunctions and eigenvalues are obtained and discussed in Sec. IV. The quasilinear particle and electron heat fluxes are given in Sec. V, where recent experimental results obtained on the Japan Atomic Energy Research Institute Tokamak 60 Upgrade (JT-60U) (Ref. 3) are also discussed. Summary, conclusions, and comments on the ballooning limit of the electron drift branch and on shear damping in cylindrical plasmas are the subjects of Sec. VI.

\section{GYROKINETIC EQUATION}

In Ref. 4, modes elongated along the direction of the magnetic field are represented by

$$
\begin{aligned}
\Phi(\psi, \chi, \varphi)= & \sum_{l, m} \exp \left\{i l\left[\varphi-\int_{\chi_{0}}^{\chi} \nu\left(\psi_{l, m}, \chi^{\prime}\right) d \chi^{\prime}\right]\right\} \\
& \times \hat{\Phi}_{l}\left(\psi-\psi_{l, m}, \psi, \chi\right),
\end{aligned}
$$

where $\psi$ is the toroidal flux, $\chi$ a poloidal-like angle, and $\varphi$ the toroidal angle; $\nu(\psi, \chi)$ is the field line pitch angle whereas

$$
q \equiv \int_{0}^{2 \pi} \nu(\psi, \chi) d \chi / 2 \pi
$$

$q\left(\psi_{l, m}\right)=-m / l$ defines the rational surfaces; $\partial_{\left(\psi-\psi_{l, m}\right.} \hat{\Phi}_{l}$ is much larger than $\partial_{\psi} \hat{\Phi}_{l}$, the modes being radially localized; and $\partial_{\chi} \hat{\Phi}_{l} \sim 1 \ll m$ allows curvature to be taken fully into account. The gradient

$$
\begin{aligned}
\hat{n} \cdot \nabla \Phi(\psi, \chi, \varphi)= & \sum_{l, m} \exp \left\{i l\left[\varphi-\int_{\chi_{0}}^{\chi} \nu\left(\chi^{\prime}, \psi_{l, m}\right) d \chi^{\prime}\right]\right\} \\
& \times \frac{B_{\chi}}{B h_{\chi}}\left\{\partial_{\chi}+i l\left[\nu(\chi, \psi)-\nu\left(\chi, \psi_{l, m}\right)\right]\right\} \\
& \times \hat{\Phi}_{l}\left(\psi-\psi_{l, m}, \psi, \chi\right)
\end{aligned}
$$

of (2a) along the direction $\hat{n} \equiv \boldsymbol{B} / \boldsymbol{B}$ of the magnetic field is $O(1 / q R)$. Each term of the sum may be considered independently in the quasislab approximation.

We consider large aspect ratio tokamaks with nonconcentric circular magnetic surfaces and, in a first attempt to assess the role of the Shafranov shift $-\Delta_{S}(r)$, write the equations with respect to variables $r$ and $\theta$ (in lieu of $\psi$ and $\chi$ ) which define a set of circles with fixed center. Making use of the equilibrium theory of Ref. 5, it can be shown that, up to order $\epsilon$,

$h_{\chi}^{-1} \partial_{x}=r^{-1}\left(\partial_{\theta}+\Delta_{s} \sin \theta \partial_{r}\right), \quad R=R_{0}(1+\epsilon \cos \theta)$,

$B_{\chi} / B=\left\{B_{\theta, 0}+\left[\partial_{r}\left(\Delta_{S} B_{\theta, 0}\right)-\epsilon\right] \cos \theta\right\} / B_{0}$,

$1-\nu\left(\chi, \psi_{l, m}\right) / \nu(\chi, \psi)=\left(r-r_{l, m}\right)\left[\partial_{r} \ln q+\cos \theta \partial_{r}(\alpha / q)\right]$,

where $\alpha \cos \theta=\nu(r, \theta)-q(r), B_{\theta, 0}=B_{\theta, 0}(r)$, and $B_{0}, B_{\varphi, 0}$, and $R_{0}$ are constants. Without loss of generality, we set $\Delta_{S}\left(r_{l, m}\right)$ $=0$ in order to identify the circle $r_{l, m}, \theta$ with the reference rational surface; thus $\Delta_{S}(r)=\left(r-r_{l, m}\right) \Delta_{S}^{\prime}$ where $\Delta_{S}^{\prime}=\partial_{r} \Delta_{S} . \Phi$ is hereafter the electrostatic potential of the wave and $F_{e(i)}$ $=\left(2 \pi c_{e(i)}^{2}\right)^{-3 / 2} N_{e(i)} \exp \left(-v^{2} / 2 c_{e(i)}^{2}\right)$ the electron (ion) Maxwellian equilibrium distribution. The Fourier transform

$$
\begin{aligned}
\hat{g}_{e, l}^{F}\left(k_{r}, r, \chi\right)= & (2 \pi)^{-1} \int \exp \left[-i k_{r}\left(r-r_{l, m}\right)\right] \\
& \times \hat{g}_{e, l}\left(r-r_{l, m}, r, \chi\right) d r
\end{aligned}
$$

of the perturbed nonadiabatic electron distribution $\hat{g}_{e, l}=\hat{f}_{e, l}$ $+\left(e_{e} \hat{\Phi}_{l} / T_{e}\right) F_{e}$ in the vicinity of the rational surface $r=r_{l, m}$ obeys the equation

$$
\begin{aligned}
& -i \omega_{l}^{\prime} \hat{g}_{e, l}+\frac{B_{\varphi, 0}}{B_{0}} \frac{v_{\|}}{q R_{0}}\left[\left(1+\Delta_{S}^{\prime} \cos \theta\right) \partial_{\theta}+\Delta_{S}^{\prime} \sin \theta \frac{\partial}{\partial k_{r}} k_{r}\right. \\
& \left.+(1-\delta \cos \theta) \frac{\partial}{\Delta_{l} \partial k_{r}}\right] \hat{g}_{e, l} \\
& -i \frac{v_{\perp}^{2}+2 v_{\|}^{2}}{2 \Omega_{e} R_{0}} \frac{B_{\varphi, 0}}{B_{0}}\left(\frac{1}{\hat{s}_{l}} \cos \theta+k_{r} \sin \theta\right) \hat{g}_{e, l} \\
& +i\left(\omega_{l}^{\prime}-\omega_{e, l}^{*, T}\right) F_{e} \frac{e_{e} \hat{\Phi}_{l}}{T_{e}}=0,
\end{aligned}
$$

where $e_{e}$ and $\Omega_{e}=e_{e} B / m_{e}$ are the electron charge and gyrofrequency, $\omega_{l}^{\prime}$ is the wave angular frequency in the $\boldsymbol{E} \times \boldsymbol{B}$ rotating frame, $\omega_{e, l}^{* T}=\omega_{e, l}^{*}\left[1+\eta_{e}\left(v_{\perp}^{2}+v_{\|}^{2}-3 c_{e}^{2}\right) / 2 c_{e}^{2}\right], \quad \omega_{e, l}^{*}$ $=-l T_{e} \partial_{r} \ln N_{e} / e_{e} R_{0} B_{\theta, 0}$, and $\delta=\epsilon+(\alpha / q)-\left(\partial_{r} \alpha / \partial_{r} q\right)$. Assuming the orderings

$$
q \sim \hat{s} \sim 1, \quad \Delta_{S}^{\prime} \sim \epsilon \sim \mu, \quad L_{N} / R \sim \mu^{2},
$$




$$
\omega_{l}^{\prime} / \omega_{e, l}^{*} \sim 1, \quad 1 / k_{r} \Delta_{l} \sim \mu, \quad k_{r} a_{i} \sim \mu,
$$

for the equilibrium and electron drift wave $\left(\omega^{\prime} \sim \omega_{e, l}^{*}\right)$ quasislab $\left(k_{r} \Delta_{l} \gg 1, k_{r} a_{i} \sim \sqrt{\left|\hat{s} L_{N} / q R\right|}\right)$ dimensionless parameters, the terms of Eq. (3a) stand in the ratios

$$
\begin{aligned}
1: & \frac{L_{N}}{q R_{0}} \frac{\Delta_{l} \hat{s}}{a_{e}}\left\{1, \Delta_{S}^{\prime}, \Delta_{S}^{\prime}, \frac{1}{k_{r} \Delta_{l}}, \frac{\epsilon}{k_{r} \Delta_{l}}\right\} \\
& \sim\left\{\mu^{-2}, \mu^{-1}, \mu^{-1}, \mu^{-1}, 1\right\}: \frac{L_{N}}{R_{0}}\left\{1, k_{r} \Delta_{l} \hat{s}\right\} \sim\left\{\mu^{2}, \mu\right\}:\{1,1\}
\end{aligned}
$$

if, without loss of generality, we let $\hat{g}_{e, l} \sim\left(e_{e} \hat{\Phi}_{l} / T_{e}\right) F_{e}$ and $\sqrt{m_{e} / m_{i}} \sim \mu^{2} . \mu \ll 1$ is the expansion parameter.

We have verified (see Appendix A) that the poloidal angle dependence of the $\hat{n} \cdot \boldsymbol{\nabla}$ operator with $\Delta_{S}^{\prime} \sim \epsilon \sim \mu$ does not affect the results; the following simplified equation will thus be sufficient for our purpose

$$
\begin{aligned}
& -i \omega_{l}^{\prime} \hat{g}_{e, l}+\frac{B_{\varphi, 0}}{B_{0}} \frac{v_{\|}}{q R_{0}}\left(\partial_{\theta}+\frac{\partial}{\Delta_{l} \partial k_{r}}\right) \hat{g}_{e, l} \\
& -i \frac{\nu_{\perp}^{2}+2 v_{\|}^{2}}{2 \Omega_{e} R_{0}} \frac{B_{\varphi, 0}}{B_{0}}\left(\frac{1}{\hat{s} \Delta_{l}} \cos \theta+k_{r} \sin \theta\right) \hat{g}_{e, l} \\
& +i\left(\omega_{l}^{\prime}-\omega_{e, l}^{*}\right) F_{e} \frac{e_{e} \hat{\Phi}_{l}}{T_{e}}=0 .
\end{aligned}
$$

The order of magnitude of the different terms here is

$$
1:\left\{\mu^{-2}, \mu^{-1}\right\}:\left\{\mu^{2}, \mu\right\}:\{1,1\}
$$

\section{SOLUTION OF EQUATION (3b) AND EIGENVALUE EQUATION}

Equation (3b) yields in leading and first orders

$$
\begin{aligned}
& \hat{g}_{e, l}^{(0)}\left(k_{r}, \theta\right)=\hat{C}_{e, l}^{(0)}, \\
& \hat{g}_{e, l}^{(1)}=-\theta \frac{\partial \hat{C}_{e, l}^{(0)}}{\Delta_{l} \partial k_{r}}+\hat{C}_{e, l}^{(1)},
\end{aligned}
$$

where the $\hat{C}_{e, l}^{(n)}=\hat{C}_{e, l}^{(n)}\left(k_{r}\right)$ are functions of $k_{r}$ only. Along the trajectory of passing electrons, the poloidal angle $\theta$ spans the domain $]-\infty,+\infty$ [ and periodicity of $\hat{g}_{e, l}^{(1)}$ requires

$$
\left(\hat{C}_{e, l}^{(0)}\right)_{\text {pass }}=0 .
$$

Equation (7), however, provides no constraint from which to extract $\left(\hat{C}_{e, l}^{(0)}\right)_{\text {trap }}$ since, for trapped electrons, $\theta$ sways between bouncing angles $-\theta_{b}$ and $+\theta_{b}$. We define $\hat{\varphi}_{l}=e_{e} \hat{\Phi}_{l} / T_{e}$; the next order equation

$$
\begin{aligned}
& -i \omega_{l}^{\prime(0)} \hat{g}_{e, l}^{(0)}+i\left(\omega_{l}^{\prime(0)}-\omega_{e, l}^{* T}\right) F_{e} \hat{\varphi}_{l}^{(0)} \\
& +\frac{B_{\varphi, 0}}{B_{0}} \frac{v_{\|}}{q R_{0}}\left(\partial_{\theta} \hat{g}_{e, l}^{(2)}+\frac{\partial \hat{g}_{e, l}^{(1)}}{\Delta_{l} \partial k_{r}}\right)=0
\end{aligned}
$$

yields, upon dividing by $v_{\|}$and integrating over a full trapped particle orbit,

$$
\left(\hat{C}_{e, l}^{(0)}\right)_{\text {trap }}=\left(1-\frac{\omega_{e, l}^{* T}}{\omega_{l}^{\prime(0)}}\right) F_{e}\left(\oint \frac{d \theta}{v_{\|}}\right)^{-1} \oint \frac{d \theta}{v_{\|}} \hat{\varphi}_{l}^{(0)}
$$

[We have noted that

$$
\oint h(\theta) d \theta \equiv \int_{-\theta_{b}}^{+\theta_{b}} h(\theta) d \theta+\int_{+\theta_{b}}^{-\theta_{b}} h(\theta) d \theta=0
$$

for single-valued functions].

The leading-order ion distribution function is

$$
\hat{f}_{i, l}^{(0)}=-\left(\omega_{i, l}^{* T} / \omega_{l}^{\prime(0)}\right) F_{i}\left(e_{i} \hat{\Phi}_{l}^{(0)} / T_{i}\right),
$$

as usual. The charge neutrality equation thus reads

$$
\begin{aligned}
\left(1-\frac{\omega_{e, l}^{*}}{\omega_{l}^{\prime(0)}}\right) \hat{\varphi}_{l}^{(0)}= & N_{e}^{-1} \int_{\text {trapped }} d \boldsymbol{v}\left(1-\frac{\omega_{e, l}^{* T}}{\omega_{l}^{\prime(0)}}\right) \\
& \times F_{e}\left(\oint \frac{d \theta}{v_{\|}}\right)^{-1} \oint \frac{d \theta}{v_{\|}} \hat{\varphi}_{l}^{(0)} .
\end{aligned}
$$

We introduce the kinetic energy per unit mass $E=\left(v_{\perp}^{2}\right.$ $\left.+v_{\|}^{2}\right) / 2$ and the magnetic moment $\mu=v_{\perp}^{2} / 2 B$. Noting that $v_{\|}^{2}(\theta)=2(E-\mu B)=2 \epsilon E\left(\cos \theta-\cos \theta_{b}\right) /(1+\epsilon \cos \theta)$, where $\cos \theta_{b}=\left(\mu B_{0}-E\right) / \epsilon E$, we obtain

$$
\begin{aligned}
\left(\oint \frac{d \theta}{v_{\|}}\right)^{-1} \oint \frac{d \theta}{v_{\|}} \hat{\varphi}_{l}^{(0)}= & \left(\oint \frac{d \theta \sqrt{1+\epsilon \cos \theta}}{\sqrt{\cos \theta-\cos \theta_{b}}}\right)^{-1} \\
& \times \oint \frac{d \theta \sqrt{1+\epsilon \cos \theta}}{\sqrt{\cos \theta-\cos \theta_{b}}} \hat{\varphi}_{l}^{(0)} .
\end{aligned}
$$

The velocity space integral of even $v_{\|}$distribution functions over the trapped particle subspace can moreover be expressed as ${ }^{6}$

$$
\begin{aligned}
\int_{\text {trapped }} d \boldsymbol{v} \equiv & 4 \pi \int_{0}^{\infty} d E \int_{E / B(\theta=\pi)}^{E / B(\theta)} \frac{B(\theta) d \mu}{\sqrt{2[E-\mu B(\theta)]}} \\
= & 4 \pi \sqrt{\frac{\epsilon}{1+\epsilon \cos \theta}} \int_{0}^{\infty} \sqrt{E} d E \\
& \times \int_{|\theta|}^{\pi} \frac{\sin \theta_{b} d \theta_{b}}{\sqrt{2\left(\cos \theta-\cos \theta_{b}\right)}} .
\end{aligned}
$$

These equalities show that the right-hand side of the charge neutrality equation (10) is proportional to $1-\left(\omega_{e, l}^{*} / \omega_{l}^{\prime(0)}\right)$, irrespective of the functional $\hat{\varphi}_{l}^{(0)}\left(k_{r}, \theta\right)$. The frequency is thus

$$
\omega_{l}^{\prime(0)}=\omega_{e, l}^{*}
$$

at leading order. We now assume that $\partial_{\theta} \hat{\varphi}_{l}^{(0)}\left(k_{r}, \theta\right)=0$, without loss of generality; thus

$$
\left(\hat{C}_{e, l}^{(0)}\right)_{\text {trap }}=\left(1-\frac{\omega_{e, l}^{* T}}{\omega_{l}^{\prime(0)}}\right) F_{e} \hat{\varphi}_{l}^{(0)} .
$$

Equation (8) simplifies now into 


$$
\begin{aligned}
& {\left[i\left(\omega_{l}^{\prime(0)}-\omega_{e, l}^{* T}\right) F_{e} \hat{\varphi}_{l}^{(0)}+\frac{B_{\varphi, 0}}{B_{0}} \frac{v_{\|}}{q R_{0}}\left(\partial_{\theta} \hat{g}_{e, l}^{(2)}+\frac{\partial \hat{C}_{e, l}^{(1)}}{\Delta_{l} \partial k_{r}}\right)\right]_{\text {pass }}} \\
& \quad=0 \\
& \left(\hat{g}_{e, l}^{(2)}=\frac{\theta^{2}}{2} \frac{\partial^{2} \hat{C}_{e, l}^{(0)}}{\Delta_{l}^{2} \partial k_{r}^{2}}-\theta \frac{\partial \hat{C}_{e, l}^{(1)}}{\Delta_{l} \partial k_{r}}+\hat{C}_{e, l}^{(2)}\right)_{\text {trap }},
\end{aligned}
$$

respectively, for passing and trapped electrons. The function $\left(\hat{C}_{e, l}^{(1)}\right)_{\text {pass }}$ is obtained by averaging the product of Eq. $\left(8^{\prime}\right)$ by $1 / v_{\|}$over the domain $\theta \in[0-2 \pi]$, thus

$$
\frac{\partial\left(\hat{C}_{e, l}^{(1)}\right)_{\mathrm{pass}}}{\Delta_{l} \partial k_{r}}=-i\left(1-\frac{\omega_{e, l}^{* T}}{\omega_{e, l}^{*}}\right) F_{e} \frac{e_{e} \hat{\Phi}_{l}^{(0)}}{T_{e}} \frac{B_{0}}{B_{\varphi, 0}} \int_{0}^{2 \pi} \frac{q R_{0} \omega_{e, l}^{*}}{v_{\|}} \frac{d \theta}{2 \pi} .
$$

$\left(\hat{C}_{e, l}^{(1)}\right)_{\text {trap }}$ is obtained in turn by averaging over a full trapped particle orbit the product of Eq. (3b) at third order by $1 / v_{\|}$. Thus, with the help of $\left(6 b^{\prime}\right)$ and (7),

$$
\begin{aligned}
\left(\hat{C}_{e, l}^{(1)}\right)_{\text {trapped }}= & \left(1-\frac{\omega_{e, l}^{* T}}{\omega_{e, l}^{*}}\right) F_{e}\left(\oint \frac{d \theta}{v_{\|}}\right)^{-1} \oint \frac{d \theta}{v_{\|}} \hat{\varphi}_{l}^{(1)} \\
& +\frac{\omega_{l}^{(1)} \omega_{e, l}^{* T}}{\left(\omega_{e, l}^{*}\right)^{2}} F_{e} \hat{\varphi}_{l}^{(0)}
\end{aligned}
$$

(in the right-hand side, contributions proportional to $\theta$ and $\sin \theta$, arising, respectively, from substitution of $\hat{g}_{e, l}^{(1)}$ and the curvature and $\nabla B$ drifts, have integrated out because of odd parity). Neglecting, in view of the significant modulation of $v_{\|}$by the helical magnetic field, the resonant interaction between the electron drift wave and the passing electrons with small parallel velocities [represented by the Dirac function contribution in $1 / v_{\|}=P / v_{\|}+i \pi \delta\left[\left(v_{\|}-\omega_{e, l}^{*} / k_{\|}\right) \operatorname{sgn} k_{\|}\right]$, cf. Eq. (7a) and Appendix B], the first-order electron density $\hat{n}_{e l}^{(1)}$ $=\int d \boldsymbol{v} \hat{g}_{e, l}^{(1)}-N_{e} \hat{\varphi}_{l}^{(1)}$ can now be expressed as $\hat{n}_{e, l}^{(1)}$ $=\left(\omega_{l}^{\prime(1)} / \omega_{e, l}^{*}\right) \hat{\varphi}_{l}^{(0)}\left(N_{e}\right)_{\text {trap }}-\hat{\varphi}_{l}^{(1)} N_{e}$.

The equation governing the ion dynamics at first order suggests writing

$$
\begin{aligned}
\hat{g}_{i, l}^{(1)}\left(\theta, k_{r}\right)= & {\left[\hat{g}_{i, l}^{(1)}\left(k_{r}\right)\right]_{0}+\left[\hat{g}_{i, l}^{(1)}\left(k_{r}\right)\right]_{\cos } \cos \theta } \\
& +\left[\hat{g}_{i, l}^{(1)}\left(k_{r}\right)\right]_{\sin } \sin \theta, \\
\hat{\Phi}_{l}^{(1)}\left(\theta, k_{r}\right)= & {\left[\hat{\Phi}_{l}^{(1)}\left(k_{r}\right)\right]_{0}+\left[\hat{\Phi}_{l}^{(1)}\left(k_{r}\right)\right]_{\cos } \cos \theta } \\
& +\left[\hat{\Phi}_{l}^{(1)}\left(k_{r}\right)\right]_{\sin } \sin \theta,
\end{aligned}
$$

as shown in Ref. 1. Except for an odd $v_{\|}$contribution, the expressions of $\left[\hat{g}_{i, l}^{(1)}\right]_{0}$ and $\left[\hat{f}_{i, l}^{(1)}\right]_{0}$ are similar to those of $\left[\hat{g}_{i, l}^{(0)}\right]_{0}$ and $\left[\hat{f}_{i, l}^{(0)}\right]_{0}$, with $\hat{\Phi}_{l}^{(0)}$ being replaced by $\left[\hat{\Phi}_{l}^{(1)}\right]_{0}$ and $\omega_{l}^{\prime(0)}$ by $\omega_{l}^{\prime(1)}+\omega_{e, l}^{*}$. The first-order ion density $\left[\hat{n}_{i, l}^{(1)}\right]_{0}$ thus reads $\left\{-\left[\hat{\varphi}_{l}^{(1)}\right]_{0}+\left(\omega_{l}^{\prime(1)} / \omega_{e, l}^{*}\right) \hat{\varphi}_{l}^{(0)}\right\} N_{i}$ and the charge neutrality equation $e_{i}\left[\hat{n}_{i, l}^{(1)}\right]_{0}+e_{e}\left[\hat{n}_{e, l}^{(1)}\right]_{0}=0$ yields $\omega_{l}^{(1)}=0$. $\left[\hat{\Phi}_{l}^{(1)}\right]_{0}$ is left undetermined and can be absorbed in $\hat{\Phi}_{l}^{(0)}$ without loss of generality; thus $\left[\hat{\Phi}_{l}^{(1)}\right]_{0}=0$. It follows that (see Ref. 1)

$$
\left[\hat{g}_{i, l}^{(1)}\right]_{0}=-i \frac{B_{\varphi, 0}}{B_{0}} \frac{v_{\|}}{q R_{0} \omega_{e, l}^{*}} \frac{\partial}{\Delta_{l} \partial k_{r}} \hat{g}_{i, l}^{(0)} .
$$

Introducing the general form of $\left[\hat{g}_{i, l}^{(1)}\left(k_{r}\right)\right]_{\cos / \sin }$ into $e_{i}\left[\hat{n}_{i, l}^{(1)}\right]_{\cos / \sin }+e_{e}\left[n_{e, l}^{(1)}\right]_{\cos / \sin }=0$ yields the following expressions for the potentials $\left[\hat{\Phi}_{l}^{(1)}\right]_{\cos / \sin }$ :

$$
\left[\hat{\Phi}_{l}^{(1)}\right]_{\cos }=0,
$$

$$
\begin{aligned}
D\left[\hat{\Phi}_{l}^{(1)}\right]_{\sin }= & -\frac{B_{\varphi, 0}}{B_{0}} k_{r} \hat{\Phi}_{l}^{(0)} \frac{1}{N_{i}} \int d \boldsymbol{v} \\
& \times \frac{\omega_{e, l}^{*}-\omega_{i, l}^{* T}}{\omega_{e, l}^{*}+\sigma\left(v_{\|} B_{\varphi, 0} l q R_{0} B_{0}\right)} \frac{v_{\perp}^{2}+2 v_{\|}^{2}}{2 \Omega_{i} R_{0} \omega_{e, l}^{*}} F_{i},
\end{aligned}
$$

where $\sigma \equiv \pm 1$, and

$$
D=\left(1+\tau_{i}\right)-\frac{1}{N_{i}} \int d \boldsymbol{v} \frac{\omega_{e, l}^{*}-\omega_{i, l}^{* T}}{\omega_{e, l}^{*}+\sigma\left(v_{\|} B_{\varphi, 0} l q R_{0} B_{0}\right)} F_{i}
$$

(results are independent of $\sigma$ since $F_{i}$ is an even function). Equations (12a), (12b), and (13) are identical to (11a) and (11b) of Ref. 1. Furthermore, $\left[\hat{g}_{i, l}^{(1)}\left(k_{r}\right)\right]_{\sin / \mathrm{cos}}$ can be expressed as

$$
\begin{aligned}
{\left[\hat{g}_{i, l}^{(1)}\right]_{\sin }=} & \frac{1}{2}\left[\left(\omega_{e, l}^{*}+\frac{B_{\varphi, 0}}{B_{0}} \frac{v_{\|}}{q R_{0}}\right)^{-1}+\left(\omega_{e, l}^{*}-\frac{B_{\varphi, 0}}{B_{0}} \frac{v_{\|}}{q R_{0}}\right)^{-1}\right] \\
& \times\left\{\left(\omega_{e, l}^{*}-\omega_{i, l}^{* T}\right) F_{i} \frac{e_{i}\left[\hat{\Phi}_{l}^{(1)}\right]_{\sin }}{T_{i}}\right. \\
& \left.-\frac{v_{\perp}^{2}+2 v_{\|}^{2}}{2 \Omega_{i} R_{0}} \frac{B_{\varphi, 0}}{B_{0}} k_{r} \hat{g}_{i, l}^{(0)}\right\}, \\
{\left[\hat{g}_{i, l}^{(1)}\right]_{\cos }=} & \frac{i}{2}\left[\left(\omega_{e, l}^{*}+\frac{B_{\varphi, 0}}{B_{0}} \frac{v_{\|}}{q R_{0}}\right)^{-1}-\left(\omega_{e, l}^{*}-\frac{B_{\varphi, 0}}{B_{0}} \frac{v_{\|}}{q R_{0}}\right)^{-1}\right] \\
& \times\left\{\left(\omega_{e, l}^{*}-\omega_{i, l}^{* T}\right) F_{i} \frac{e_{i}\left[\hat{\Phi}_{l}^{(1)}\right]_{\sin }}{T_{i}}\right. \\
& \left.-\frac{v_{\perp}^{2}+2 v_{\|}^{2}}{2 \Omega_{i} R_{0}} \frac{B_{\varphi, 0}}{B_{0}} k_{r} \hat{g}_{i, l}^{(0)}\right\} .
\end{aligned}
$$

We note that $\left[\hat{g}_{i, l}^{(1)}\right]_{\cos }$ is odd with respect to $v_{\|}$whereas $\left[\hat{g}_{i, l}^{(1)}\right]_{\sin }$ is even. Also

$$
\left(\hat{C}_{e, l}^{(1)}\right)_{\text {trapped }}=0,
$$

as a consequence of (12a).

The radial eigenvalue equation is obtained in next order from the $\theta$-averaged charge neutrality equation $\left[n_{e, l}^{(2)}\right]_{0}$ $=\left[n_{i, l}^{(2)}\right]_{0}$. For passing electrons, it follows from $\left(8^{\prime}\right)$ that $\left[\hat{g}_{e, l}^{(2)}\right]\left(\theta, k_{r}\right)-\hat{C}_{e, l}^{(2)}\left(k_{r}\right)$ is odd with respect to $v_{\|}$; thus $\int d \boldsymbol{v} \hat{g}_{e, l}^{(2)}$ $=\int d \boldsymbol{v} \hat{C}_{e, l}^{(2)}$. The function $\hat{C}_{e, l}^{(2)}\left(k_{r}\right)$ is determined by averaging the product of the third-order equation by $v_{\|}^{-1}$ over the domain $\theta \in[0-2 \pi]$. Only the $v_{\|}$even terms contribute to the perturbed density $\hat{n}_{e, l}^{(2)}$. Those are given by 


$$
\left[\frac{\partial^{2}\left(\hat{C}_{e, l}^{(2)}\right)_{e \mathrm{ven}}}{\Delta_{l}^{2} \partial k_{r}^{2}}=\left(1-\frac{\omega_{e, l}^{*} T}{\omega_{e, l}^{*}}\right) F_{e}\left(\int_{0}^{2 \pi} \frac{q R_{0} \omega_{e, l}^{*}}{v_{\|}} \frac{d \theta}{2 \pi}\right)^{2}\right]_{\mathrm{pass}}
$$

For trapped electrons, $\hat{C}_{e, l}^{(0)}$ is proportional to $\left(\omega_{e, l}^{*}-\omega_{e, l}^{* T}\right)$ and $\hat{C}_{e, l}^{(1)}=0$. It follows from $\left(8^{\prime \prime}\right)$ that $\hat{g}_{e, l}^{(2)}-\hat{C}_{e, l}^{(2)}$ is also proportional to $\left(\omega_{e, l}^{*}-\omega_{e, l}^{* T}\right)$ and does not contribute to $\hat{n}_{e, l}^{(2)}$. Multiplying the fourth-order equation by $v_{\|}^{-1}$ and integrating over a trapped particle orbit yields

$$
\begin{aligned}
\left\{\hat{C}_{e, l}^{(2)}=\right. & \frac{\omega_{l}^{\prime(2)} \omega_{e, l}^{* T}}{\left(\omega_{e, l}^{*}\right)^{2}} F_{e} \hat{\varphi}_{l}^{(0)}-\left(\oint \frac{d \theta}{v_{\|}}\right)^{-1} \oint \frac{d \theta}{v_{\|}}\left[\left(\hat{g}_{e, l}^{(2)}-\hat{C}_{e, l}^{(2)}\right)\right. \\
& \left.-\left(1-\frac{\omega_{e, l}^{* T}}{\omega_{e, l}^{*}}\right) F_{e} \hat{\varphi}_{l}^{(2)}\right] \\
& -\left(\oint \frac{d \theta}{v_{\|}}\right)^{-1} \frac{B_{\varphi, 0}}{B_{0}} \oint \frac{d \theta}{v_{\|}} \frac{v_{\perp}^{2}+2 v_{\|}^{2}}{2 \Omega_{e} R_{0} \omega_{e, l}^{*}}\left(\frac{1}{\hat{s} \Delta_{l}} \cos \theta \hat{g}_{e, l}^{(0)}\right. \\
& \left.\left.+k_{r} \sin \theta \hat{g}_{e, l}^{(1)}\right)\right\}_{\text {trap }} .
\end{aligned}
$$

The second-order perturbed electron density is thus

$$
\begin{aligned}
\hat{n}_{e, l}^{(2)}= & \int d \boldsymbol{v}\left[\left(\hat{g}_{e, l}^{(2)}\right)_{\text {pass }}+\left(\hat{g}_{e, l}^{(2)}\right)_{\text {trap }}\right]-\hat{\varphi}_{l}^{(2)} N_{e} \\
= & -\hat{\varphi}_{l}^{(2)} N_{e}+\frac{\omega_{l}^{\prime(2)}}{\omega_{e, l}^{*}} \hat{\varphi}_{l}^{(0)}\left(N_{e}\right)_{\text {trap }} \\
& +\int d \boldsymbol{v}\left(\hat{C}_{e, l}^{(1)}+\hat{C}_{e, l}^{(2)}\right)_{\text {pass }}-\int d \boldsymbol{v}\left(\oint \frac{d \theta}{v_{\|}}\right)^{-1} \frac{B_{\varphi, 0}}{B_{0}} \\
& \times \oint \frac{d \theta}{v_{\|}} \frac{v_{\perp}^{2}+2 v_{\|}^{2}}{2 \Omega_{e} R_{0} \omega_{e, l}^{*} \Delta_{l}}\left(\hat{s}^{-1} \cos \theta-\theta \sin \theta \frac{k_{r} \partial}{\partial k_{r}}\right) \hat{g}_{e, l}^{(0)} .
\end{aligned}
$$

We shall neglect, in the following, the nonadiabatic contribution of passing electrons (its order of magnitude is discussed in Appendix B). Equation (14) thus yields, upon approximating $v_{\perp}^{2}+2 v_{\|}^{2} \cong 2 E$ for trapped electrons,

$$
\begin{aligned}
\frac{\hat{n}_{e, l}^{(2)}}{N_{e}}= & -\hat{\varphi}_{l}^{(2)}+\frac{\omega_{l}^{\prime(2)}}{\omega_{e, l}^{*}} \hat{\varphi}_{l}^{(0)}\left(f_{e}\right)_{\text {trap }}+\frac{3 \sqrt{2 \epsilon} \eta_{e} q}{4} \\
& \times \frac{L_{N}}{L_{S}}\left(\hat{s}^{-1} \mathfrak{I}_{0}-\Im_{1} \frac{k_{r} \partial}{\partial k_{r}}\right) \hat{\varphi}_{l}^{(0)},
\end{aligned}
$$

where $L_{S}=q R / \hat{s}$ is the magnetic shear length,

$$
\left(f_{e}\right)_{\text {trap }}=\frac{\left(N_{e}\right)_{\text {trap }}}{N_{e}}=\sqrt{1-\frac{B(\theta)}{B(\theta=\pi)}} \cong \sqrt{\epsilon(1+\cos \theta)},
$$

and

$$
\begin{aligned}
\mathfrak{I}_{0 ; 1}(\theta)= & \int_{|\theta|}^{\pi} \frac{\sin \theta_{b} d \theta_{b}}{\sqrt{2\left(\cos \theta-\cos \theta_{b}\right)}} \\
& \times\left(\int_{-\theta_{b}}^{\theta_{b}} \frac{d \theta^{\prime}}{\sqrt{\cos \theta^{\prime}-\cos \theta_{b}}}\right)^{-1} \\
& \times \int_{-\theta_{b}}^{\theta_{b}} \frac{\left(\cos \theta^{\prime} ; \theta^{\prime \prime} \sin \theta^{\prime}\right) d \theta^{\prime \prime}}{\sqrt{\cos \theta^{\prime \prime}-\cos \theta_{b}}}
\end{aligned}
$$

$\left(\Im_{1}\right.$ is positive since $\left.|\theta|<\pi\right)$.

Extracting now $\left[\hat{g}_{i, l}^{(2)}\right]_{0}$ from the equation

$$
\begin{gathered}
-i \omega_{e, l}^{*}\left[\hat{g}_{i, l}^{(2)}\right]_{0}-i \omega_{l}^{\prime(2)} \hat{g}_{i, l}^{(0)}-i \frac{v_{\perp}^{2}+2 v_{\|}^{2}}{4 \Omega_{i} R_{0}} \frac{B_{\varphi, 0}}{B_{0}} k_{r}\left[\hat{g}_{i, l}^{(1)}\right]_{\mathrm{sin}} \\
+\frac{B_{\varphi, 0}}{B_{0}} \frac{v_{\|}}{q R_{0}} \frac{\partial}{\Delta_{l} \partial k_{r}}\left[\hat{g}_{i, l}^{(1)}\right]_{0}+i\left[( \omega _ { e , l } ^ { * } - \omega _ { i , l } ^ { * T } ) \left(\frac{e_{i}\left[\hat{\Phi}_{l}^{(2)}\right]_{0}}{T_{i}}\right.\right. \\
\left.\left.-\frac{k_{r}^{2} v_{\perp}^{2}}{4 \Omega_{i}^{2}} \frac{e_{i} \hat{\Phi}_{l}^{(0)}}{T_{i}}\right)+\omega_{i, l}^{\prime}(2) \frac{e_{i} \hat{\Phi}_{l}^{(0)}}{T_{i}}\right] F_{i}=0
\end{gathered}
$$

leads to

$$
\begin{aligned}
{\left[\hat{n}_{i, l}^{(2)}\right]_{0}=} & \int d \boldsymbol{v}\left\{\left[\hat{g}_{i, l}^{(2)}\right]_{0}-\frac{k_{r}^{2} v_{\perp}^{2}}{4 \Omega_{i}^{2}} \hat{g}_{i, l}^{(0)}\right\}-\frac{e_{i}\left[\hat{\Phi}_{l}^{(2)}\right]_{0}}{T_{i}} N_{i} \\
= & \tau_{i}\left(\frac{e_{i}\left[\hat{\Phi}_{l}^{(2)}\right]_{0}}{T_{i}}-\frac{\omega_{l}^{(2)}}{\omega_{e, l}^{*}} \frac{e_{i} \hat{\Phi}_{l}^{(0)}}{T_{i}}\right) N_{i} \\
& -\frac{\partial^{2}}{\Delta_{l}^{2} \partial k_{r}^{2}} \int d \boldsymbol{v} \frac{v_{\|}^{2}}{\left(q R_{0} \omega_{e, l}^{*}\right)^{2}} \hat{g}_{i, l}^{(0)} \\
& -\int d \boldsymbol{v} \frac{k_{r}^{2} v_{\perp}^{2}}{2 \Omega_{i}^{2}}\left(1-\frac{\omega_{i, l}^{*} T}{\omega_{e, l}^{*}}\right) F_{i} \frac{e_{i} \hat{\Phi}_{l}^{(0)}}{T_{i}} \\
& -\frac{B_{\varphi, 0}}{B_{0}} \int d \boldsymbol{v} \frac{k_{r}\left(v_{\perp}^{2}+2 v_{\|}^{2}\right)}{4 \Omega_{i} R_{0} \omega_{e, l}^{*}}\left[\hat{g}_{i, l}^{(1)}\right]_{\sin },
\end{aligned}
$$

where one recognizes, respectively, in the second, third, and last terms the contributions from parallel ion motion, finite Larmor radius, and $\nabla B$ and curvature drifts; the latter differentiate the toroidal from the cylindrical geometry ion dynamics.

\section{EIGENFUNCTIONS AND EIGENVALUES}

In Ref. 1, we considered the ion response in the two limiting cases where the velocity integrals can be approximated either by their principal parts or by their residues. The latter are hereafter treated as corrections, assuming

$$
\begin{aligned}
& c_{i} / q R_{0} \omega_{e, l}^{*} \ll 1, \\
& \lambda_{i}=\left(q R_{0} \omega_{e, l}^{*} / c_{i}\right)^{5} \exp \left[-\left(q R_{0} \omega_{e, l}^{*}\right)^{2} / 2 c_{i}^{2}\right] \ll 1 .
\end{aligned}
$$

Thus the approximations 


$$
\begin{aligned}
& D \cong-\alpha_{i}\left(\frac{c_{i}}{q R_{0} \omega_{e, l}^{*}}\right)^{2}\left(1-i \sqrt{\frac{\pi}{8}} \frac{\tau_{i} \eta_{i} \lambda_{i}}{\alpha_{i}} \operatorname{sgn} q\right), \\
& {\left[\hat{\Phi}_{l}^{(1)}\right]_{\sin } \cong \frac{q R_{0} \omega_{e, l}^{*}}{c_{i}} \frac{B_{\varphi, 0}}{B_{0}} q k_{r} a_{i} \hat{\Phi}_{l}^{(0)}(2} \\
& \left.+i \sqrt{\frac{\pi}{8}} \frac{\tau_{i} \eta_{i} \lambda_{i}}{\alpha_{i}} \operatorname{sgn} q\right), \\
& {\left[\hat{g}_{i, l}^{(1)}\right]_{\sin } \cong \frac{q R_{0} \omega_{e, l}^{*}}{c_{i}} \frac{B_{\varphi, 0}}{B_{0}} q k_{r} a_{i} \frac{e_{i} \hat{\Phi}_{l}^{(0)}}{T_{i}}} \\
& \times\left[\left(2+i \sqrt{\frac{\pi}{8}} \frac{\tau_{i} \eta_{i} \lambda_{i}}{\alpha_{i}} \operatorname{sgn} q\right)\right. \\
& \left.-\frac{i \pi}{2} \frac{q R_{0} \omega_{e, l}^{*}}{c_{i}} \operatorname{sgn} q \sum_{ \pm} \delta\left(\frac{v_{\|}}{c_{i}} \pm \frac{q R_{0} \omega_{e, l}^{*}}{c_{i}} \frac{B_{\varphi, 0}}{B_{0}}\right)\right] \\
& \times\left(1-\frac{\omega_{i, l}^{*} T}{\omega_{e, l}^{*}}\right) F_{i}, \\
& \frac{\left[n_{i, l}^{(2)}\right]_{0}}{N_{i}}=\tau_{i}\left(\frac{e_{i}\left[\hat{\Phi}_{l}^{(2)}\right]_{0}}{T_{i}}-\frac{\omega_{l}^{(2)}}{\omega_{e, l}^{*}} \frac{e_{i}}{\hat{\Phi}_{l}^{(0)}}\right) \\
& -\alpha_{i} \tau_{i}^{2} \frac{L_{N}^{2}}{L_{S}^{2}} \frac{\partial^{2}}{\partial\left(k_{r} a_{i}\right)^{2}} \frac{e_{i} \hat{\Phi}_{l}^{(0)}}{T_{i}}-\alpha_{i} k_{r}^{2} a_{i}^{2}\left[1+2 q^{2}\right. \\
& \left.\times\left(1+i \sqrt{\frac{\pi}{8}} \frac{\tau_{i} \eta_{i} \lambda_{i}}{4 \alpha_{i}} \operatorname{sgn} q\right)\right] \frac{e_{i} \hat{\Phi}_{l}^{(0)}}{T_{i}},
\end{aligned}
$$

where $\operatorname{sgn} x=x /|x|$.

Charge neutrality leads accordingly to the eigenvalue equation

$$
\begin{aligned}
\left\{\varpi_{l}\right. & +\frac{\partial^{2}}{\partial \hat{k}_{r}^{2}}+\left[1+2 q^{2}\left(1+i \delta_{i} \operatorname{sgn} q\right)\right] \hat{k}_{r}^{2}+\operatorname{sgn}\left(L_{N} / L_{S}\right) \\
& \left.\times\left(-\hat{s}^{-1} \delta_{0}+\delta_{1} \frac{\hat{k}_{r} \partial}{\partial \hat{k}_{r}}\right)\right\} \hat{\varphi}_{l}^{(0)}=0,
\end{aligned}
$$

where

$$
\begin{aligned}
& \varpi_{l}=\left[1-\left\langle\left(f_{e}\right)_{\text {trap }}\right\rangle_{\theta}\right] \frac{\left|L_{S}\right|}{\left|L_{N}\right|} \frac{\omega_{l}^{(2)}}{\alpha_{i} \omega_{e, l}^{*}}, \\
& \hat{k}_{r}^{2}=\frac{\left|L_{s}\right|}{\left|L_{N}\right|} k_{r}^{2} a_{s}^{2}, \quad a_{s}^{2}=a_{i}^{2} \tau_{i}^{-1}, \\
& \delta_{0 ; 1}=\frac{3 \sqrt{2 \epsilon} \eta_{e} q}{4 \alpha_{i}}\left\langle\Im_{0 ; 1}\right\rangle_{\theta}, \\
& \delta_{i}=\sqrt{\frac{\pi}{8}} \frac{\tau_{i} \eta_{i} \lambda_{i}}{4 \alpha_{i}},
\end{aligned}
$$

and $\langle\cdots\rangle_{\theta}$ stands for $\int_{0}^{2 \pi} \cdots d \theta / 2 \pi$. The set of orthogonal solutions and eigenvalues are

$$
\begin{aligned}
& \hat{\varphi}_{l, n}^{(0)}=\exp \left(-\xi \hat{k}_{r}^{2} / 2\right) H_{n}\left[\hat{k}_{r} \sqrt{\xi-0.5 \delta_{1} \operatorname{sgn}\left(L_{N} / L_{S}\right)}\right], \\
& \varpi_{l, n}=(2 n+1) \xi+\left(\hat{s}^{-1} \delta_{0}-n \delta_{1}\right) \operatorname{sgn}\left(L_{N} / L_{S}\right),
\end{aligned}
$$

where the $H_{n}$ 's are Hermite polynomials ${ }^{7}$ and

$$
\xi^{2}-\delta_{1} \operatorname{sgn}\left(L_{N} / L_{S}\right) \xi+\left[1+2 q^{2}\left(1+i \delta_{i} \operatorname{sgn} q\right)\right]=0 .
$$

Noting that $\left(\delta_{1}\right)^{2}$ is usually smaller than $4\left(1+2 q^{2}\right), \xi$ may be approximated by [cf. Eqs. (19b), (20c), and (20d)]

$$
\xi=\frac{\left|\delta_{1}\right|}{2} \operatorname{sgn}\left(\hat{s} L_{T_{e}}\right) \mp \frac{q^{2}\left|\delta_{i}\right|}{\sqrt{1+2 q^{2}}} \operatorname{sgn}\left(\eta_{i} \omega_{e, l}^{*}\right) \pm i \sqrt{1+2 q^{2}}
$$

(we assume $\alpha_{i}>0$ ). The growth/decay rate is thus

$$
\gamma_{l, n}=\Im m \omega_{l}^{\prime(2)}= \pm(2 n+1) \sqrt{1+2 q^{2}} \frac{\left|L_{N}\right|}{\left|L_{S}\right|} \frac{\alpha_{i} \omega_{e, l}^{*}}{1-\left\langle\left(f_{e}\right)_{\text {trap }}\right\rangle_{\theta}} .
$$

Instability (damping) requires accordingly that $\pm \omega_{e, l}^{*}$ be positive (negative), viz., $\omega_{e, l}^{*}>0\left(\omega_{e, l}^{*}<0\right)$ if the upper sign is chosen and $\omega_{e, l}^{*}<0\left(\omega_{e, l}^{*}>0\right)$ if, instead, the lower sign is selected. Since the inverse Fourier transform of the fundamental solution is

$$
\begin{array}{r}
\hat{\varphi}_{l, n=0}^{(0)}(\hat{x}, t) \propto \exp \left(-\frac{\hat{x}^{2}}{2 \xi}-i \omega t\right) \cong \exp \left[-\frac{\hat{x}^{2} \operatorname{Re} \xi}{2\left(1+2 q^{2}\right)}\right. \\
\left.+\gamma_{l, n=0} t\right] \exp \left[ \pm i \frac{\hat{x}^{2}}{2 \sqrt{1+2 q^{2}}}-i \omega_{e, l}^{*} t\right]
\end{array}
$$

(where $\hat{x}^{2}=\left|L_{N} / L_{s}\right|\left(r-r_{l, m}\right)^{2} / a_{s}^{2}$ ), bounded unstable solutions are outgoing disturbances, propagating away from the rational surface, and bounded damped solutions propagate towards that surface. Two cases must be envisaged:

(i) $\left|\delta_{1}\right|<2 q^{2}\left|\delta_{i}\right| / \sqrt{1+2 q^{2}}$ : according to Eqs. (23') and (24), spatial localization $(\operatorname{Re} \xi>0)$ implies damping if $\eta_{i}$ is positive and growth if $\eta_{i}$ is negative (a similar result has been obtained earlier in the framework of a local theory ${ }^{8}$ ).

(ii) $\left|\delta_{1}\right|>2 q^{2}\left|\delta_{i}\right| / \sqrt{1+2 q^{2}}$ : here, spatial localization requests that $\operatorname{sgn} L_{T_{e}}=\operatorname{sgn} \hat{s}$; according to Eq. (24), the trapped electron response introduces a pair of growing and damped solutions. There is no physically meaningful (bounded) solution if $\operatorname{sgn} L_{T_{e}}$ $=-\operatorname{sgn} \hat{s}$.

The collisionless instabilities just described are thus driven by magnetic shear under unusual conditions, either $\eta_{i}<0$ (reversed ion temperature and density gradients) or $\operatorname{sgn} L_{T_{e}}=\operatorname{sgn} \hat{s}$ (parallel electron temperature and safety factor gradients, i.e., negative magnetic shear if $L_{T_{e}}<0$ as is usually the case), and their growth rate increases for the higher-order Hermite polynomial eigenfunctions. The situation in (ii) is particularly reminiscent of that encountered with the ion temperature gradient mode in the quasislab approximation: a damped/growing pair of solutions with $\gamma_{l, n}$ $\propto(2 n+1)|\hat{s}|$, cf. Eq. (91) in Ref. 9 .

Electron collisions have not been taken into account in the present work. According to a simplified theory, ${ }^{10}$ they are 
destabilizing if $\left|\omega_{e, l}^{*}\right|<3 \nu_{e, \text { eff }}$, where $\nu_{e, \text { eff }}=\nu_{e} / \epsilon$ is the effective trapped electron collision frequency, and stabilizing otherwise. The condition of weak resonant interaction of ions with the sidebands, $c_{i} / q R_{0} \omega_{e, l}^{*}<O(1)$, for potentially unstable $\left(\omega_{e, l}^{*}<3 v_{e, \text { eff }}\right)$ collision-driven modes is thus

$$
\begin{aligned}
c_{i} / q R_{0} \nu_{e, \text { eff }} \cong & 2 \epsilon \sqrt{\tau_{i} / A_{i}}\left(T_{e} / 10^{3} \mathrm{eV}\right)^{2} / \\
& q R_{0}(m) Z_{\mathrm{eff}}\left(N_{e} / 10^{20} \mathrm{~m}^{-3}\right)<O(3),
\end{aligned}
$$

where $A_{i}$ is the ion atomic mass.

\section{QUASILINEAR PARTICLE AND ELECTRON HEAT FLUXES}

The quasilinear expressions for the magnetic surface averaged particle and electron heat fluxes $\Gamma_{e, i}$ $=\Sigma\left\langle i l \phi n_{e, i}^{*}\right\rangle_{\theta} / R_{0} B_{\theta, 0}$ and $Q_{e}=\Sigma\left\langle i l \phi p_{e}^{*}\right\rangle_{\theta} / R_{0} B_{\theta, 0}$ are

$$
\frac{\Gamma_{e, i}}{N_{e}}=\frac{\alpha_{i} T_{e}}{\left|e_{e} B_{\varphi, 0}\right| r} \frac{-L_{N}}{R_{0}} \frac{\sqrt{1+2 q^{2}}}{1-\left\langle\left(f_{e}\right)_{\text {trap }}\right\rangle_{\theta}}|\hat{s}| \sum_{l, n}|l|(2 n+1)\left|\bar{\varphi}_{l, n}\right|^{2},
$$

$$
\begin{aligned}
\frac{Q_{e}}{P_{e}}= & \frac{2 \alpha_{i} T_{e}}{\left|e_{e} B_{\varphi, 0}\right| r} \frac{-L_{N}}{R_{0}}\left(\frac{q R_{0} \omega_{e, l}^{*}}{c_{s}}\right)^{2} \frac{\left\langle H_{3}+2\left(H_{2}-H_{1}\right)\right\rangle_{\theta}}{\left\langle\Im_{1}\right\rangle_{\theta}} \\
& \times\left.\sqrt{1+2 q^{2}}|\hat{s}| \sum_{l, n}|l|(2 n+1) \overline{\mid \varphi_{l, n}}\right|^{2}
\end{aligned}
$$

where $p_{e}$ is the electron pressure perturbation, the star refers to the complex conjugate,

$$
\begin{aligned}
\left.\overline{\mid \varphi_{l, n}}\right|^{2} & =\left|\Delta_{l}\right|^{-1} \int_{-\infty}^{\infty} d x\left|\varphi_{l, n}^{(0)}\left(x=r-r_{l, m}, r\right)\right|^{2} \\
& =\left|\Delta_{l}\right|^{-1} 2 \pi \int_{-\infty}^{\infty} d k_{r}\left|\varphi_{l, n}^{(0)}\left(k_{r}, r\right)\right|^{2}
\end{aligned}
$$

is the spatial average in the neighborhood of $r$ of the turbulence level for the $n$th Hermite polynomial solution,

$$
\begin{aligned}
& H_{1 ; 3}(\theta)=(\cos \theta ; \theta \sin \theta) \int_{|\theta|}^{\pi} h\left(\theta_{b}\right) d \theta_{b}, \\
& H_{2}(\theta)=\int_{|\theta|}^{\pi}\left[h\left(\theta_{b}\right)\left(\int_{-\theta_{b}}^{\theta_{b}} \frac{d \theta}{v_{\|}}\right)^{-1}\left(\int_{-\theta_{b}}^{\theta_{b}} \frac{d \theta}{v_{\|}} \cos \theta\right)\right] d \theta_{b},
\end{aligned}
$$

and $h\left(\theta_{b}\right)=\sin \theta_{b} / \sqrt{2\left(\cos \theta-\cos \theta_{b}\right)} \quad$ (demonstration and derivation of the full ion and electron energy equations are left to a forthcoming paper). The unusually large ratio $Q_{e} / T_{e} \Gamma_{e, i} \approx\left(q R_{0} \omega_{e, l}^{*} / c_{s}\right)^{2}$ is a consequence of the unusually large pressure perturbation which, as the potential $\left[\hat{\Phi}_{l}^{(1)}\right]_{\mathrm{sin}}$, arises in conjunction with the sidebands, see $\left(12 b^{\prime}\right)$ and (19a). This result, which is by no means restricted to collisionless instabilities, has largely been ignored in the literature as emphasis has usually been laid on the ballooning limit and formalism. ${ }^{11}$
Worth noting is also that the spatial average of the turbulence is proportional to the absolute value of the magnetic shear parameter $|\hat{s}|$ as the unstable modes are separated by $\left|\Delta_{l}\right|=1 /\left|l \partial_{r} q\right|$. This is one of the reasons why internal transport barriers (ITB) form near flux surfaces where magnetic shear is weak.

Inequality (26) is satisfied out of the electron internal transport barrier $\left(T_{e}<6 \mathrm{keV}, N_{e}<4 \times 10^{18} \mathrm{~m}^{-3}, R_{0}=3.4 \mathrm{~m}\right.$, $\epsilon>0.11, q>2.2, \hat{s}>0)$ obtained on the JT-60U tokamak ${ }^{3,12}$ when electron cyclotron heating and current drive $(\mathrm{ECH} / \mathrm{ECCD})$ is applied to a lower hybrid current drive (LHCD) reversed shear (RS) deuterium plasma $\left(\sqrt{\tau_{i}} / Z_{\text {eff }}\right.$ is clearly smaller than unity); in this region, collisional destabilization of the trapped electron mode (TEM) thus exceeds ion Landau damping. By contrast, the requirement of weak Landau damping leads to $\omega_{e, l}^{*} / \nu_{e, \text { eff }}>O(3)$ inside of the ITB and particularly at the top of the pedestal $\left(6 \mathrm{keV}<T_{e}\right.$ $<26 \mathrm{keV}, \quad 4.5 \times 10^{18} \mathrm{~m}^{-3}>N_{e}>4 \times 10^{18} \mathrm{~m}^{-3}, \quad \epsilon<0.11, \quad q$ $>2.2, \hat{s}<0$ ), corresponding to collisional damping of the TEM; however, the reverse magnetic shear instability discussed in Sec. IV is now relevant and its growth rate easily exceeds the low collisional damping rate. The predicted large ratio $Q_{e} / T_{e} \Gamma_{e, i}$ and the qualitative behavior of the TEM growth rate through the discharge may explain the reported "box-type" electron temperature profile displaying strong flattening in the region of large negative $\hat{s}$, cf. Figs. 10 and 12 in Ref. 12.

\section{SUMMARY, CONCLUSIONS, AND COMMENTS}

We have derived the eigenvalue equation for the collisionless quasislab electron drift branch, keeping the trapped electron response and the toroidal ion dynamics fully into account. The theory predicts instability if either $\eta_{i}<0$ or $\hat{s} L_{T_{e}}>0$ (i.e., $\hat{s}<0$ if, as usual, $L_{T_{e}}<0$ ), depending on the value of $\left|\delta_{1}\right| \sqrt{1+2 q^{2}} / 2 q^{2}\left|\delta_{i}\right|$, see the discussion following Eq. (25); the growth rate is proportional to the absolute value of the magnetic shear parameter, see Eq. (24), and the unstable collisionless solutions have a radiating character. The presence of sidebands driven by the ion dynamics greatly enhances the electron heat flux. An application of the theory to a negative central shear JT-60U discharge has been discussed. The theory is also reactor relevant.

We have focused on the quasislab sub-branch of the electron drift wave (or trapped electron mode). A ballooning toroidal sub-branch may coexist if it verifies the inequality $\left|k_{r} \Delta_{l}\right|<1$. This assumption leads to the following radial eigenvalue equation [see Eq. (7) in Ref. 13 and Eq. (80) in Ref. 14]:

$$
\left[\bar{D} a_{s}^{2} \frac{\partial^{2}}{\partial x^{2}}-\frac{L_{N}^{2}}{L_{S}^{2}}\left(\frac{\omega_{e, l}^{*}}{\omega_{l}^{(0)}}\right)^{2} \frac{x^{2}}{a_{s}^{2}}+\alpha_{i} \frac{\omega_{l}^{\prime(2)}}{\omega_{l}^{\prime(0)}}\right] \hat{\varphi}=\alpha_{i}\left(\frac{\hat{n}_{e}}{N_{e}}+\hat{\varphi}\right),
$$

where

$$
\bar{D}=-\cos \theta_{0}\left(\Delta_{l} / a_{S}\right)^{2}(1-2 \hat{s}) L_{N} / R_{0}-\left(1+2 q^{2}\right),
$$

$\omega_{l}^{\prime(0)}$ is the leading order frequency and $\theta_{0}$ the ballooning angle. [There is a change in the sign of $\cos \theta_{0}$ with respect to 
Ref. 13 as $\theta=0$ corresponded there to the inboard equatorial plane; $-\left(1+2 q^{2}\right)$ has been introduced in an ad hoc manner in Eq. $\left(30^{\prime}\right)$, instead of -1 , in order to make the bridge with the quasislab branch discussed in this paper.] Assuming adiabatic electrons, the fundamental solution of (30) reads

$$
\begin{aligned}
& \hat{\varphi}=\exp \frac{\mp x^{2}}{2 \sqrt{\bar{D}} a_{s}^{2}} \frac{\left|L_{N}\right|}{\left|L_{S}\right|} \frac{\omega_{e, l}^{*}}{\omega_{l}^{\prime(0)},} \\
& \frac{\omega^{\prime(2)}}{\omega_{e, l}^{*}}= \pm \alpha_{i}^{-1} \sqrt{\bar{D}} \frac{\left|L_{N}\right|}{\left|L_{S}\right|} .
\end{aligned}
$$

The cases $\bar{D}>0$ and $\bar{D}<0$ are discussed separately below.

If $\bar{D}$ is positive and we define $\sqrt{\bar{D}}>0$, then only the upper sign solution (31) is bounded since $\omega_{l}^{\prime(0)}=\omega_{e, l}^{*}$. Furthermore, $\bar{D}>0$ results in a mere upper frequency shift as $\omega_{l}^{\prime(2)}$ is real. Comparing $\left\langle x^{2}\right\rangle=\int x^{2} \hat{\varphi} d x / \int \hat{\varphi} d x=a_{s}^{2} \sqrt{\bar{D}}\left|L_{S} / L_{N}\right|$ to $\Delta_{l}^{2}$, the ballooning mode requirement $\left\langle\left(k_{r} \Delta_{l}\right)^{2}\right\rangle^{-1}=\left\langle\left(x / \Delta_{l}\right)^{2}\right\rangle=\alpha_{1}$ $\gg 1$ leads to

$$
|1-2 \hat{s}| \frac{q^{2} a_{s}^{2}}{\Delta_{l}^{2} \hat{s}^{2}} \frac{R_{0}}{\left|L_{N}\right|}>\left(\alpha_{2}\right)^{2},
$$

where $\alpha_{2}>\alpha_{1}$. The assumption $\bar{D}>0$ demands further that

$$
\frac{\Delta_{l}^{2}}{a_{s}^{2}}|1-2 \hat{s}| \frac{\left|L_{N}\right|}{R_{0}}>\left(1+2 q^{2}\right) .
$$

Inequalities (32) and (33) are compatible if

$$
\left|\hat{s}^{-1}-2\right|>\alpha_{2} \sqrt{q^{-2}+2}
$$

or approximately

$$
|\hat{s}|<1 / \alpha_{2} \sqrt{2} \ll 1 .
$$

Condition (34b) is generally not satisfied in tokamaks. The strong ballooning limit of the electron drift branch is thus irrelevant, except perhaps for weak magnetic shear [it must, however, be verified by introducing an appropriate ordering that Eq. (30) still holds in that limit]. We should add that for $\hat{s}<1 / 2, \cos \theta_{0}>0$ is a necessary condition for $\bar{D}>0$ if, as usual, $L_{N}<0$; thus the modes would be localized on the outboard midplane where the trapped electron population is largest. For $\hat{s}>1 / 2$, however, they should be localized on the inboard midplane where the trapped electron population is negligible!

If $\bar{D}$ is negative, then the two solutions (31) are oscillating in space. Defining $\sqrt{\bar{D}} \equiv i \sqrt{|\bar{D}|}$ and replacing $\omega_{l}^{\prime(0)}$ by $\omega_{e, l}^{*}+i \gamma$ with $\gamma>0$ to ensure causality, we find that $\mp \omega_{e, l}^{*}$ must be positive for an asymptotically bounded solution. $\Im m \omega_{l}^{\prime(2)}$ is thus negative, i.e., the mode is damped due to magnetic shear. The above causality argument, which is also followed in cylindrical geometry, ${ }^{15}$ can, however, be fallacious as it relies on a higher-order result, the growth rate, introduced on the same footing as the leading-order frequency without verifying that other corrections of the same order would not modify the asymptotic character of the solution.

\section{ACKNOWLEDGMENTS}

Valuable discussions with Professor P. K. Kaw are gratefully acknowledged.

The work of one of the authors (R.S.) was partially financed by the Bundesministerium für Bildung und Forschung in the framework of the cooperation agreement between the governments of the Republic of India and the Federal Republic of Germany in the fields of Scientific and Technology Development (Project No. IND 01/012).

\section{APPENDIX A: NEGLIGIBLE ROLE OF SHAFRANOV SHIFT IF $\Delta_{S}^{\prime} \sim \epsilon$}

Equation (12a) is to be modified as follows:

$$
\left[\hat{\Phi}_{l}^{(1)}\right]_{\cos }=\Delta_{S}^{\prime} \frac{\partial\left(k_{r} \hat{\Phi}_{l}^{(0)}\right)}{\partial k_{r}},
$$

if the Shafranov shift $\Delta_{S}^{\prime} \sim \epsilon \sim \mu$ is taken into account. Remembering that the gyrokinetic equation (3) is written in cylindrical coordinates, the interpretation of (A1) is that the perturbed potential is constant on the flux surface of equation $s=r+\Delta_{S} \cos \theta . \quad \hat{\Phi}\left(k_{r}\right)+\Delta_{S}^{\prime} \cos \theta \partial\left[k_{r} \hat{\Phi}\left(k_{r}\right)\right] / \partial k_{r} \equiv \hat{\Phi}_{l}^{(0)}$ $+\left[\hat{\Phi}_{l}^{(1)}\right]_{\cos } \cos \theta$ is indeed the Fourier transform of $\hat{\Phi}(s)$ $=\hat{\Phi}(r)+\Delta_{S} \cos \theta \partial_{r} \hat{\Phi}(r)$ if $\Delta_{S}\left(r_{l, m}\right)=0$ by convention. Other results are not modified by the Shafranov shift with the present ordering.

\section{APPENDIX B: REQUIREMENT FOR NEGLECTING THE NONADIABATIC PASSING ELECTRON RESPONSE}

$\int d \boldsymbol{v} \hat{C}_{e, l}^{(2)}$ is smaller than the last term of Eq. (14) if, pass. approximately, $k_{r}^{2} a_{e}^{2}<L_{N}^{3} / R_{0} L_{S}^{2}$. Keeping into account that resonant interaction of barely passing electrons with the waves is reduced by the modulation of $v_{\|}$from $v_{\|} \cong 0$ to $v_{\|}$ $\sim \sqrt{\epsilon c_{e}}$, owing to the variation of the magnetic field strength, it can be shown that $\int d \boldsymbol{v} \hat{C}_{e, l}^{(1)}$ is also negligible if $k_{r}^{2} a_{e}^{2}<L_{N}^{3} \sqrt{\epsilon} / R_{0} L_{S}^{2}$.

${ }^{1}$ A. L. Rogister, Phys. Plasmas 2, 2729 (1995).

${ }^{2}$ J. W. Connor and R. J. Hastie, Plasma Phys. Controlled Fusion 46, 1501 (2004).

${ }^{3}$ H. Ninomiya and the JT-60U Team, Phys. Fluids B 4, 2070 (1992).

${ }^{4}$ A. L. Rogister and G. Hasselberg, Plasma Phys. Controlled Fusion 27, 193 (1985).

${ }^{5}$ V. D. Shafranov, Plasma Equilibrium in a Magnetic Field, in Reviews of Plasma Physics, Vol. 2, edited by M. A. Leontovich (Consultants Bureau, New York, 1966), p. 103.

${ }^{6}$ E. A. Frieman, Phys. Fluids 13, 490 (1970).

${ }^{7}$ M. Abramowitz and I. A. Stegun, Handbook of Mathematical Functions (Dover, New York, 1965), Chap. 22.

${ }^{8}$ A. L. Rogister, R. Singh, and P. K. Kaw, Phys. Plasmas 11, 2106 (2004).

${ }^{9}$ A. L. Rogister, Phys. Plasmas 7, 5070 (2000); see also A. L. Rogister, Nucl. Fusion 41, 1101 (2001).

${ }^{10}$ W. M. Manheimer and C. N. Lashmore-Davies, MHD and Microinstabilities in Confined Plasmas (Institute of Physics, Bristol, 1989), Chaps. 1819.

${ }^{11}$ J. W. Connor, J. R. Hastie, and J. B. Taylor, Phys. Rev. Lett. 40, 396 (1978); J. W. Connor, J. R. Hastie, and J. B. Taylor, Proc. R. Soc. London, Ser. A 365, 1 (1979).

${ }^{12}$ Y. Ikeda, T. Suzuki, A. Isayama, K. Kajiwara, S. Ide, T. Ozeki, S. Moriyama, M. Seki, T. Fujita, M. Takechi, Y. Koide, T. Fukuda, H. Tak- 
enaga, O. Naito, K. Hamamatsu, T. Hatae, T. Fujii, and JT-60U team, in Proceedings of the 12th Joint Workshop on Electron Cyclotron Emission and Electron Cyclotron Heating, Aix-en-Provence, 2002 (World Scientific, Singapore, 2002), p. 247; http://wshop.free.fr/ec12/PAPERS/021Ikeda.pdf
${ }^{13}$ G. Hasselberg and A. L. Rogister, Plasma Phys. 22, 805 (1980). ${ }^{14}$ W. M. Tang, Nucl. Fusion 18, 1089 (1978).

${ }^{15}$ L. D. Pearlstein and H. L. Berk, Phys. Rev. Lett. 23, 220 (1969). 